\title{
BMD and fracture risk in T2DM-clarifying a paradox
}

The prevalence of osteoporosis and vertebral fractures is high in postmenopausal women with type 2 diabetes mellitus (T2DM), independent of blood glucose control, report Maíra Viégas and colleagues in the Journal of Diabetes and its Complications. In another study, published in Osteoporosis International, Mishaela Rubin and her team show that BMD in postmenopausal women with and without T2DM does not differ, but that the levels of biochemical markers of bone turnover are lower in women with T2DM.

T2DM is associated with an increased risk of fractures, but the explanation for this association is unclear, as patients with T2DM have been reported to have normal or above normal BMD.

Viégas et al. studied a group of 148 postmenopausal women with T2DM. The prevalence of osteoporosis, assessed by dual-energy X-ray absorptiometry (DXA), was $30.4 \%$ at the lumbar spine and $9.5 \%$ at the femoral neck. The prevalence of vertebral fractures was 23\%. Age $>60$ years and osteoporosis were associated with increased likelihood of fractures, irrespective of blood glucose control. Patients with vertebral fractures had lower absolute BMD at the lumbar spine and femoral neck than patients without fractures. These findings support the idea that increased bone loss in the setting of T2DM might contribute to increased fracture risk. "There are grounds for considering risk factors other than blood glucose control to prevent bone fragility in postmenopausal women with T2DM," write the researchers, who highlight the need for prospective studies aimed at clarifying the risk factors for osteoporotic fractures related to T2DM.

"We became interested in whether bone is more fragile in T2DM in a way that isn't being detected by conventional BMD testing," says Mishaela Rubin, whose team compared bone structure measured by DXA and high-resolution peripheral quantitative CT (HR-pQCT) in 25 postmenopausal women with T2DM and 25 matched controls. The researchers found that bone structure was not different in the two groups (with the exception of the cortical area of the tibia). However, the levels of two markers of bone turnover, P1NP and osteocalcin, were lower in the group of women with T2DM. Low bone turnover could lead to an increased risk of fractures.

"Our next step will be to investigate biopsies taken from the hip bone of women with T2DM to see whether the collagen is coated with sugar," says Rubin, "which might weaken the bone in a way

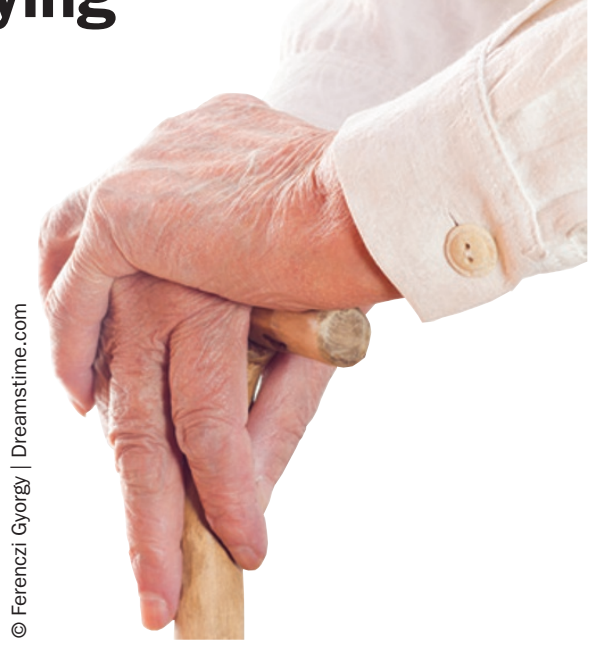

that wouldn't be detected by usual bone density testing."

Rubin stresses that patients with T2DM should be aware of bone health issues, such as the importance of calcium and vitamin D supplementation and prevention of falls.

Joana Osório 